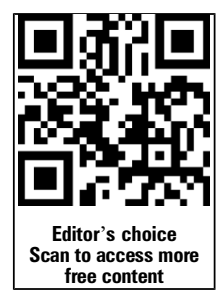

${ }^{1}$ Institute of Bioengineering and Nanotechnology,

Singapore

${ }^{2}$ Department of Pathology, Singapore General Hospital, Singapore

${ }^{3}$ Department of Anatomy, Yong Loo Lin School of Medicine, National University of Singapore, Singapore ${ }^{4}$ Department of Physiology, Yong Loo Lin School of Medicine, National University of Singapore, Singapore

${ }^{5}$ Mechanobiology Institute, National University of Singapore, Singapore ${ }^{6}$ Singapore-MIT Alliance for Research and Technology, Singapore

Duke-NUS Graduate Medical School, Singapore

\section{Correspondence to} Dr Min-Han Tan,

Institute of Bioengineering and Nanotechnology, 31 Biopolis Way, The Nanos, \#04-01,138669, Singapore; mhtan@ibn.a-star.edu.sg or Dr Puay Hoon Tan, Department of Pathology, Singapore General Hospital, 20 College Road, Academia, Level 7, Diagnostics Tower, 169856, Singapore; tan.puay.hoon@sgh.com.sg

Received 26 June 2015 Accepted 5 July 2015 Published Online First 24 July 2015

\title{
Second harmonic generation microscopy is a novel technique for differential diagnosis of breast fibroepithelial lesions
}

Wai Jin Tan, ${ }^{1}$ Jie Yan, ${ }^{1}$ Shuoyu $\mathrm{Xu}_{1}{ }^{1}$ Aye Aye Thike, ${ }^{2}$ Boon Huat Bay, ${ }^{3}$ Hanry $\mathrm{Yu},{ }^{1}, 4,5,6$ Min-Han Tan, ${ }^{1}$ Puay Hoon $\operatorname{Tan}^{2,3,7}$

\section{ABSTRACT}

Breast fibroepithelial lesions, including fibroadenomas and phyllodes tumours, are commonly encountered in clinical practice. As histological differences between these two related entities may be subtle, resulting in a challenging differential diagnosis, pathological techniques to assist the differential diagnosis of these two entities are of high interest. An accurate diagnosis at biopsy is important given corresponding implications for clinical decision-making including surgical extent and monitoring. Second harmonic generation (SHG) microscopy is a recently developed optical imaging technique capable of robust, powerful and unbiased label-free direct detection of collagen fibril structure in tissue without the use of antibodies. We constructed tissue microarrays emulating limited materials on biopsy to investigate quantitative collagen signal in fibroepithelial lesions using SHG microscopy. Archived formalin-fixed paraffin-embedded materials of 47 fibroepithelial lesions (14 fibroadenomas and 33 phyllodes tumours) were evaluated. Higher collagen signal on SHG microscopy was observed in fibroadenomas than phyllodes tumours on SHG imaging ( $p<0.001$, area under the curve 0.859). At an automated threshold (2.5 million positive pixels), the sensitivity and specificity of the SHG microscopy for fibroadenoma classification was $71.4 \%$ and $84.4 \%$, respectively. To corroborate these findings, we performed immunohistochemistry on tissue array sections using collagen I and III primary antibodies. Both collagen I and III immunohistochemical expressions were also significantly higher in fibroadenomas than in phyllodes tumours $(p<0.001)$. In conclusion, label-free collagen quantitation on SHG microscopy is a novel imaging approach that can aid the differential diagnosis of fibroepithelial lesions.

\section{FINDINGS}

Second harmonic generation (SHG) microscopy is a recently developed optical imaging technique capable of robust and powerful label-free detection of collagen fibril structure in tissue. ${ }^{1}$ Recently, its feasibility in quantitatively assessing collagen signals in small biopsies has been demonstrated. ${ }^{2}$ Specifically in breast cancer, SHG-detected tumouraligned collagen signature was shown to be a prognostic signature for survival. ${ }^{3}$ Fibroepithelial lesions are commonly encountered in breast biopsies, on which differential diagnoses of the more common fibroadenomas and the rarer phyllodes tumours are considered. Similar radiological and occasional overlapping histological features of both entities may pose challenges in distinguishing them on limited material of core biopsies. Fibroadenomas are managed conservatively while phyllodes tumours are usually excised due to their greater tendency to recur. We anticipated that SHG microscopy could have utility in aiding diagnosis in this setting based on an anticipated increase in collagen signals in fibroadenomas. To assess this on limited material, we constructed tissue microarrays with a $2 \mathrm{~mm}$ diameter core from archival formalin-fixed paraffin-embedded tissue of 14 fibroadenomas and 33 phyllodes tumours (19 benign, 10 borderline and 4 malignant) diagnosed at the Department of Pathology, Singapore General Hospital with ethics approval from the Centralised Institutional Review Board (CIRB 2005/002/F). An unstained tissue microarray section of $4 \mu \mathrm{m}$ was subjected to SHG microscopic scanning as previously described. ${ }^{4}$ Raw images were processed based on the Otsu segmentation method and amount of collagen pixels expressed in green colour was quantified for respective spots of the tissue microarray. We observed strikingly higher collagen signals on SHG imaging in fibroadenomas than phyllodes tumours with median pixels of 1.26 million and 3.14 million, respectively (figure $1 \mathrm{~A}$ ). The area under the curve by receiver operating characteristic (ROC) analysis was $0.859(\mathrm{p}<0.001)$. Based on a threshold value (2.5 million pixels) calculated from the ROC analysis, the sensitivity and specificity of the SHG microscopy for fibroadenoma classification was $71.4 \%$ and $84.4 \%$, respectively (figure 1C). To corroborate these findings, we stained the tissue array sections with collagen I and III antibodies by immunohistochemistry and semiquantitatively assessed both the intensity and percentage of staining using the immunoreactive scoring (IRS) method. ${ }^{5}$ Both collagen I and III immunohistochemical expressions were significantly higher in fibroadenomas than in phyllodes tumours (figure 1A), with a median difference of IRS $67.5(\mathrm{p}<0.001)$ and $47.5(\mathrm{p}<0.001)$, respectively, substantiating our observations on the SHG microscopy platform. Furthermore, significant correlations were observed between collagen signals quantified from the SHG platform with collagen I $(\mathrm{r}=0.412, \mathrm{p}=0.005)$ and collagen III $(\mathrm{r}=0.640$, $\mathrm{p}<0.001)$ immunohistochemical expression. Recent development of SHG microscopy for in vivo imaging technique ${ }^{67}$ suggests its potential utility as a non-invasive, label-free diagnostic tool in breast fibroepithelial lesions. Stromal collagen differences between fibroadenomas and phyllodes tumours may also provide further insight into their biological disparity. 

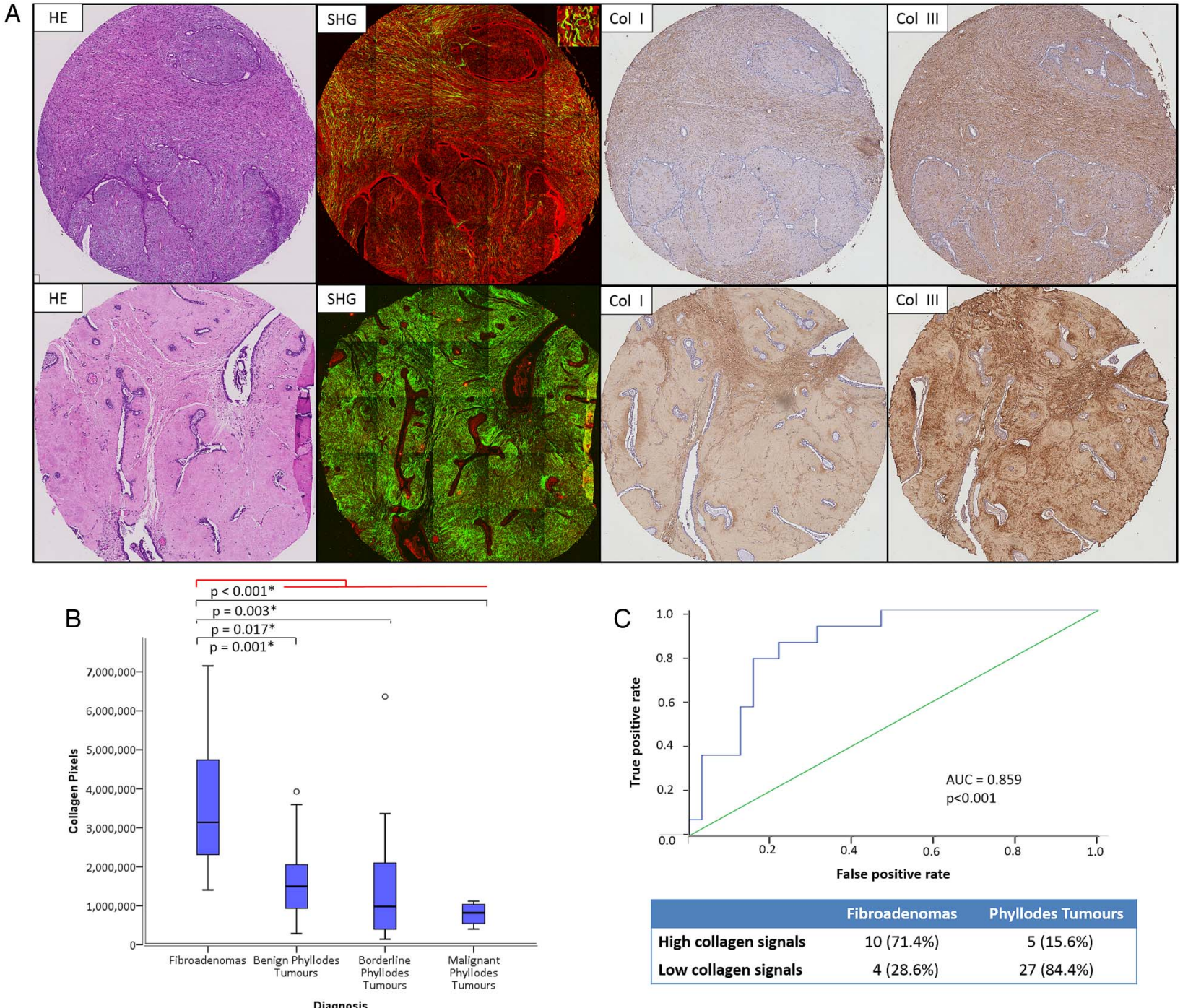

Figure 1 Expression of collagen is higher in fibroadenomas than phyllodes tumours. (A) Representative example of a phyllodes tumour (upper panel) and a fibroadenoma (lower panel) with corresponding images of H\&E stain, second harmonic generation (SHG), collagen I immunohistochemical expression (Col I) and collagen III immunohistochemical expression (Col III). Collagen (green) signals on SHG are clearly higher in the fibroadenoma. (B) Boxplot of collagen signals in fibroadenomas and phyllodes tumours (stratified into three groups). Expression of collagen was significantly higher in fibroadenomas across four analyses using the Mann-Whitney $U$ test. (C) Receiver operating characteristic curve differentiating fibroadenomas and phyllodes tumours with SHG collagen signals. Area under the curve (AUC) is 0.859 with $p$ value $<0.001$. A significant proportion of fibroadenomas expressed high collagen signals (defined with above 2.5 million pixels).

\section{Take home messages}

- Second-harmonic generation microscopy is a label-free optical imaging technology which can detect and quantitate collagen in tissue sections without immunostaining.

- Higher collagen signals are observed in breast fibroadenomas in comparison to phyllodes tumours, allowing for useful discrimination.

\section{Handling editor Cheok Soon Lee}

Acknowledgements This study was supported by the Institute of Bioengineering and Nanotechnology (Biomedical Research Council, Agency for Science, Technology and Research, Singapore).
Contributors WJT collated data, constructed tissue microarrays, performed immunohistochemistry and analysed the results and drafted the manuscript. JY and SX performed the SHG scanning and data analysis. AAT collected samples and validated immunohistochemical scoring. BBH contributed to the scientific content of the study. HY provided support for SHG scanning. M-HT designed the study, supervised data analysis, drafted the manuscript and coordinated the study. PHT participated in the design of the study, provided study material and drafted the manuscript.

Funding Institute of Bioengineering and Nanotechnology (Biomedical Research Council, Agency for Science, Technology and Research, Singapore).

Competing interests None declared.

Ethics approval Centralised Institutional Review Board.

Provenance and peer review Not commissioned; externally peer reviewed.

\section{REFERENCES}

1 Chen X, Nadiarynkh O, Plotnikov S, et al. Second harmonic generation microscopy for quantitative analysis of collagen fibrillar structure. Nat Protoc 2012;7:654-69. 
2 Ambekar R, Lau TY, Walsh M, et al. Quantifying collagen structure in breast biopsies using second-harmonic generation imaging. Biomed Opt Express 2012;3: 2021-35.

3 Conklin MW, Eickhoff JC, Riching KM, et al. Aligned collagen is a prognostic signature for survival in human breast carcinoma. Am J Pathol 2011;178: 1221-32.

4 He Y, Kang $\mathrm{CH}$, Xu S, et al. Toward surface quantification of liver fibrosis progression. J Biomed Opt 2010;15:056007.
5 Tan WJ, Thike AA, Bay BH, et al. Immunohistochemical expression of homeoproteins Six 1 and Pax3 in breast phyllodes tumours correlates with histological grade and clinical outcome. Histopathology 2014;64:807-17.

6 Wu PC, Hsieh TY, Tsai ZU, et al. In vivo quantification of the structural changes of collagens in a melanoma microenvironment with second and third harmonic generation microscopy. Sci Rep 2015;5:8879.

7 Wu X, Chen G, Lu J, et al. Label-free detection of breast masses using multiphoton microscopy. PLOS ONE 2013;8:e65933. 